\title{
A Weinreb Nitrile Oxide and Nitrone for Cycloadditions
}

\author{
Ajit Kumar Parhi, Richard W. Franck* \\ Department of Chemistry, Hunter College of The City University of New York, 695 Park \\ Avenue, New York, NY 10021 \\ Email: rfranck@hunter.cuny.edu, Fax: 212-772-5332.
}

\section{Supporting Information}

\section{Table of Contents}

Instruments and Materials........................................ 2

Experimental procedures and compound characterization data ............3

NMR and Mass Spectra.............................................. 7 


\section{Instruments and Materials}

NMR spectra were recorded at $300 \mathrm{MHz}$ or $500 \mathrm{MHz}\left({ }^{1} \mathrm{H}\right)$ and $75 \mathrm{MHz}\left({ }^{13} \mathrm{C}\right)$ in deuterated chloroform. The assignment of proton and carbon NMR peaks was supported by routine COSY and for some cases by NOESY spectra.

Electrospray ionization (ESI) mass spectra experiments were performed by Dr. Clifford E. Soll at the Hunter College Mass Spectrometry Facility. Typical ESI method: solvent: 1/1 acetonitrile/water $+0.1 \%$ HOAc $+50 \mu \mathrm{L} \mathrm{NH} 4 \mathrm{Ac}$, flow: $0.50 \mathrm{~mL} / \mathrm{min}$, positive ion mode, fragmentor voltage: $30-200 \mathrm{~V}$, drying gas at $175^{\circ} \mathrm{C}$.

All air-moisture sensitive reactions were performed under a positive pressure of dry nitrogen. All solvents and reagents were purified prior to use according to standard laboratory procedures. Low temperatures were recorded as bath temperatures.

Thin layer chromatography analyses were carried out on precoated aluminium sheets of silica gel $60 \mathrm{~F}$ 254. UV light and vanillin, potassium permanganate or phosphomolybdic acid spray was used to visualize the components on the TLC plates.

Flash column chromatography was carried out with silica gel 60 (230-400 mesh), using ACS reagent grade petroleum ether, ethyl acetate. 


\section{Experimental procedures and compound characterization data}

\section{Weinreb amide-aldehyde 8}

Ozone was bubbled through a solution of cinnamate $7(2.2 \mathrm{~g}, 11.5 \mathrm{mmol})$ in 1:1 $\mathrm{CH}_{2} \mathrm{Cl}_{2}-\mathrm{MeOH}(80 \mathrm{~mL})$ at $-78{ }^{0} \mathrm{C}$ for $5 \mathrm{~h}$. After persistence of the blue color of the solution, the excess of $\mathrm{O}_{3}$ was removed with a stream of $\mathrm{O}_{2}$. Nitrogen was then bubbled into the mixture until the blue color disappeared. $\mathrm{Me}_{2} \mathrm{~S}(1.7 \mathrm{~mL}$, $23.04 \mathrm{mmol}$ ) was added, and the solution was allowed to stir at room temperature overnight. The solvent was removed in vacuum and the residue was purified by column chromatography on silica gel (EtOAc / PE, 2:3) to give the product (1.2 g , 78\%). ${ }^{1} \mathrm{H}$ NMR: (300 $\left.\mathrm{MHz}, \mathrm{CDCl}_{3}\right) \delta 5.22(1 \mathrm{H}, \mathrm{d}, \mathrm{J}=10.9 \mathrm{~Hz}), 4.38$ $(1 \mathrm{H}, \mathrm{d}, \mathrm{J}=10.9 \mathrm{~Hz}), 3.77(3 \mathrm{H}, \mathrm{s}), 3.48(3 \mathrm{H}, \mathrm{s}), 3.26(3 \mathrm{H}, \mathrm{s})$; ESI MS (calcd for $\left.\mathrm{C}_{5} \mathrm{H}_{11} \mathrm{NO}_{4}, 149\right) \mathrm{m} / z: 150$ $\left(\mathrm{M}+\mathrm{H}^{+}\right)$.

\section{General method for cycloaddition}

To a solution of Weinreb amide functionalized aldehyde $8(2.06 \mathrm{mmol})$ in $7 \mathrm{~mL}$ of $1: 1$ mixture of $\mathrm{H}_{2} \mathrm{O}$ and ether was added hydroxylamine hydrochloride $\left(357 \mathrm{mg}, 5.1 \mathrm{mmol}, 2.5\right.$ equiv.) and $\mathrm{NaHCO}_{3}(519$ $\mathrm{mg}$ ). The mixture was stirred for $30 \mathrm{~min}$ and then extracted with $\mathrm{CH}_{2} \mathrm{Cl}_{2}$ three times. The combined extract was dried with $\mathrm{Na}_{2} \mathrm{SO}_{4}$, filtered and concentrated to give crude 9. A solution of crude 9 (360 mg, $2.7 \mathrm{mmol}$ in $2 \mathrm{~mL}$ of $\mathrm{CHCl}_{3}$ ) was added to a suspension of $\mathrm{NCS}(545 \mathrm{mg}, 4 \mathrm{mmol})$ in $2 \mathrm{~mL}$ of anhydrous $\mathrm{CHCl}_{3}$ and pyridine $(50 \mu \mathrm{L})$ at room temperature. The completion of chlorination was observed by the disappearance of suspended NCS (about $30 \mathrm{~min}$ ). The reaction mixture was concentrated in vacuum and co-evaporated with toluene. Dipolarophiles (2 equiv) was added to an ice-cold stirred solution of $\mathbf{1 0}$ in 2 $\mathrm{mL}$ of $\mathrm{CH}_{2} \mathrm{Cl}_{2}$. After stirring for $10 \mathrm{~min}$ at $0{ }^{0} \mathrm{C}$, a solution of triethylamine $(150 \mu \mathrm{L})$ in $1 \mathrm{~mL} \mathrm{of} \mathrm{CH}_{2} \mathrm{Cl}_{2}$ is added drop wise over a $3 \mathrm{~h}$ period, maintaining the temperature between $1^{0} \mathrm{C}$ to $4{ }^{0} \mathrm{C}$. The reaction mixture was stirred for an additional $20 \mathrm{~min}$ at $0{ }^{0} \mathrm{C}$, filtered to remove the triethylamine hydrochloride, which was washed with ether. The combined filtrate was washed with brine, then aqueous $\mathrm{HCl}$, dried, filtered and concentrated to give the crude product which was subjected to flash column chromatography to give pure product in 50-60\% yield. (Potassium permanganate spray was used to visualize the components on the TLC plates). 


\section{Cycloaddition with Propargyl bromide. 11 (entry-1, table-1)}

${ }^{1} \mathrm{H}$ NMR: $\left(300 \mathrm{MHz}, \mathrm{CDCl}_{3}\right) \delta 6.66(1 \mathrm{H}, \mathrm{s}), 4.66(1 \mathrm{H}, \mathrm{s}), 4.5(1 \mathrm{H}, \mathrm{s}), 3.79(3 \mathrm{H}, \mathrm{s}), 3.38(3 \mathrm{H}, \mathrm{brs}) ;{ }^{13} \mathrm{C}$ : NMR (75 MHz, $\left.\mathrm{CDCl}_{3}\right) \delta 167.8,158.2,104.8,62.3,34.2,33.4$ (br); ESI MS (calcd for $\mathrm{C}_{7} \mathrm{H}_{9} \mathrm{BrN}_{2} \mathrm{O}_{3}, 248$ ) $m / z: 266.0,267.9\left(\mathrm{M}+\mathrm{NH}_{4}^{+}\right)$.

\section{Cycloaddition with Propargyl alcohol. 12 (entry-2, table-1)}

${ }^{1} \mathrm{H}$ NMR: $\left(300 \mathrm{MHz}, \mathrm{CDCl}_{3}\right) \delta 6.53(1 \mathrm{H}, \mathrm{s}), 4.78(2 \mathrm{H}, \mathrm{s}), 3.76(3 \mathrm{H}, \mathrm{s}), 3.38$ (3H, brs). ${ }^{13} \mathrm{C}$ NMR: $(75$ $\left.\mathrm{MHz}, \mathrm{CDCl}_{3}\right) \delta 172.3,160.8(\mathrm{br}), 157.6,102.8,62.3,56.5,33.2(\mathrm{br}) ; \mathrm{ESI} \mathrm{MS}$ (calcd for $\mathrm{C}_{7} \mathrm{H}_{10} \mathrm{~N}_{2} \mathrm{O}_{4}, 186$ ) $m / z: 204.1\left(\mathrm{M}+\mathrm{NH}_{4}^{+}\right)$.

\section{Cycloaddition with Phenyl propyne. 13 (entry-3, table-1)}

${ }^{1} \mathrm{H}$ NMR: (300 MHz, $\left.\mathrm{CDCl}_{3}\right) \delta$ 7.35-7.24 (5H, m), 6.27 (1H, s), 4.11 (2H, s), 3.77 (3H, s), 3.37 (3H, brs). ${ }^{13} \mathrm{C}$ NMR: $\left(75 \mathrm{MHz}, \mathrm{CDCl}_{3}\right) \delta 172.5,158.0,135.6,130.0,128.9,127.4,103.1,62.2,33.4$ (br), 33.3; ESI MS (calcd for $\left.\mathrm{C}_{13} \mathrm{H}_{14} \mathrm{~N}_{2} \mathrm{O}_{3}, 246\right) \mathrm{m} / z: 264.1\left(\mathrm{M}+\mathrm{NH}_{4}^{+}\right)$.

\section{Cycloaddition with 1-Phenyl-2-propyn-1-ol. 14 (entry-4, table-1)}

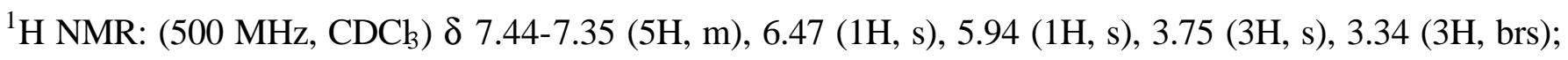

${ }^{13} \mathrm{C}$ : NMR (75 MHz, $\left.\mathrm{CDCl}_{3}\right) \delta 174.4,160.6(\mathrm{br}), 157.5,139.6,128.8,128.7,126.7,102.8,69.4,62.2$, 33.8(br); ESI MS (calcd for $\left.\mathrm{C}_{13} \mathrm{H}_{14} \mathrm{~N}_{2} \mathrm{O}_{4}, 262\right) \mathrm{m} / z: 280\left(\mathrm{M}+\mathrm{NH}_{4}{ }^{+}\right)$.

\section{Cycloaddition with ethyl acrylate. 16 (entry-6, table-1)}

${ }^{1} \mathrm{H}$ NMR: $\left(500 \mathrm{MHz}, \mathrm{CDCl}_{3}\right) \delta 5.03(1 \mathrm{H}, \mathrm{dd}, \mathrm{J}=7 \mathrm{~Hz}, \mathrm{~J}=11.5 \mathrm{~Hz}), 4.17(2 \mathrm{H}, \mathrm{qt}, \mathrm{J}=7 \mathrm{~Hz}) 3.69(3 \mathrm{H}, \mathrm{s}), 3.6-$ $3.4(2 \mathrm{H}, \mathrm{m}), 3.23(3 \mathrm{H}, \mathrm{brs}), 1.23(3 \mathrm{H}, \mathrm{t}, \mathrm{J}=7 \mathrm{~Hz}) ;{ }^{13} \mathrm{C} \mathrm{NMR}:\left(75 \mathrm{MHz}, \mathrm{CDCl}_{3}\right) \delta 168.9,160.1(\mathrm{br}), 151.7$, 77.8, 61.9, 61.8, 39.8, 33.4(br), 13.9; ESI MS (calcd for $\left.\mathrm{C}_{9} \mathrm{H}_{14} \mathrm{~N}_{2} \mathrm{O}_{5}, 230\right) \mathrm{m} / z: 248.1\left(\mathrm{M}+\mathrm{NH}_{4}{ }^{+}\right.$).

\section{WA nitrone and subsequent cycloaddition.}

To a solution of Weinreb amide functionalized aldehyde $8(185 \mathrm{mg}, 1.2 \mathrm{mmol})$ in $4 \mathrm{~mL}$ of 1:1 mixture of $\mathrm{H}_{2} \mathrm{O}$ and ether was added benzyl hydroxylamine hydrochloride (250 mg, $1.6 \mathrm{mmol}, 1.3$ equiv) and 
$\mathrm{NaHCO}_{3}(320 \mathrm{mg})$. The mixture was stirred for $30 \mathrm{~min}$ and then extracted with $\mathrm{CH}_{2} \mathrm{Cl}_{2}$ three times. The

combined extract was dried with $\mathrm{Na}_{2} \mathrm{SO}_{4}$, filtered and concentrated to give crude $\mathbf{5}$. N-benzyl nitrone 5 $(1.7 \mathrm{mmol})$ was dissolved in an excess of ethyl vinyl ether or ethyl acrylate $(37 \mathrm{mmol})$. The reaction mixture was heated at $80^{\circ} \mathrm{C}$ for two days. After cooling, the excess solvent was removed by evaporation under high vacuum. The resulting reaction mixture was chromatographed to give product.

\section{Cycloaddition with ethyl vinyl ether. 17 (entry-1, table-2)}

${ }^{1} \mathrm{H}$ NMR: (500 MHz, CDCl$) \delta$ 7.45-7.20 (5H, m), $5.36(1 \mathrm{H}, \mathrm{d}, \mathrm{J}=4 \mathrm{~Hz}), 4.41(2 \mathrm{H}, \mathrm{d}, \mathrm{J}=13 \mathrm{~Hz}), 4.15$ (1H, d, J=12.5Hz), $3.73(1 \mathrm{H}, \mathrm{m}), 3.48-3.43(4 \mathrm{H}, \mathrm{m}), 3.11(3 \mathrm{H}, \mathrm{s}), 2.93(1 \mathrm{H}, \mathrm{m}), 2.55-2.51(1 \mathrm{H}, \mathrm{m}), 1.2(3 \mathrm{H}, \mathrm{t})$; ${ }^{13} \mathrm{C}$ NMR: $\left(75 \mathrm{MHz}, \mathrm{CDCl}_{3}\right) \delta 171.2,137.6,129.4,128.3,127.4,105.7,64.5,64.0,63.2,61.1,38.3$, 32.8,15.3; ESI MS (calcd for $\left.\mathrm{C}_{15} \mathrm{H}_{22} \mathrm{~N}_{2} \mathrm{O}_{4}, 294\right) \mathrm{m} / z: 295.1\left(\mathrm{M}+\mathrm{H}^{+}\right.$).

\section{Cycloaddition with ethyl acrylate. 18 (entry-2, table-2)}

${ }^{1} \mathrm{H}$ NMR: (500 MHz, $\left.\mathrm{CDCl}_{3}\right) \delta$ 7.42-7.25 (5H, m), $4.77(1 \mathrm{H}, \mathrm{t}, \mathrm{J}=8.5 \mathrm{~Hz}), 4.37(1 \mathrm{H}, \mathrm{m}), 4.27-4.20(3 \mathrm{H}$, m), $3.92(1 \mathrm{H}, \mathrm{d}, \mathrm{J}=13 \mathrm{~Hz}), 3.41(3 \mathrm{H}, \mathrm{s}), 3.12(3 \mathrm{H}, \mathrm{s}), 2.95(1 \mathrm{H}, \mathrm{m}), 2.71-2.65(1 \mathrm{H}, \mathrm{m}), 1.29(3 \mathrm{H}, \mathrm{t}$, $\mathrm{J}=7 \mathrm{~Hz}) ;{ }^{13} \mathrm{C}$ NMR: $\left(75 \mathrm{MHz}, \mathrm{CDCl}_{3}\right) \delta(172.2,170.2,136.7,129.7,128.3,127.5,77.8,63.8,62.7,61.4$, 61.2, 34.6, 32.6, 14.3; ESI MS (calcd for $\left.\mathrm{C}_{15} \mathrm{H}_{22} \mathrm{~N}_{2} \mathrm{O}_{4}, 322\right) \mathrm{m} / z: 323.1\left(\mathrm{M}+\mathrm{H}^{+}\right)$.

\section{Isoxazole ketone 19}

To a solution of 13 (entry-3, Table-1) (156 mg, $0.634 \mathrm{mmol})$ in $5 \mathrm{~mL}$ of anhydrous THF was added 4 equiv of methyl magnesium bromide $(2.6 \mathrm{mmol})$ at $0{ }^{0} \mathrm{C}$. The reaction mixture was stirred at $0{ }^{0} \mathrm{C}$ until TLC showed no starting amide. The reaction mixture was poured into $5 \% \mathrm{HCl}$ in ethanol at $0{ }^{0} \mathrm{C}$ and the mixture was partitioned between brine and a 1:1 mixture of ether and $\mathrm{CH}_{2} \mathrm{Cl}_{2}$. The organic extract was dried with $\mathrm{Na}_{2} \mathrm{SO}_{4}$ and evaporated in vacuum. The concentrate was purified by column chromatography (silica gel, $25 \%$ EtOAc / petroleum ether) to provide $125 \mathrm{mg}$ of the product. ${ }^{1} \mathrm{H}$ NMR: $\left(300 \mathrm{MHz}, \mathrm{CDCl}_{3}\right) \delta$ 7.36-7.23 (5H, m), $6.30(1 \mathrm{H}, \mathrm{s}), 4.11(2 \mathrm{H}, \mathrm{s}), 2.61(3 \mathrm{H}, \mathrm{s}) ;{ }^{13} \mathrm{C} \mathrm{NMR:}(75 \mathrm{MHz}$, $\left.\mathrm{CDCl}_{3}\right) \delta 192.1,174.0,162.4,135.5,129.1,128.9,127.5,100.6,33.5,27.4$; ESI MS (calcd for $\left.\mathrm{C}_{12} \mathrm{H}_{11} \mathrm{NO}_{2}, 201\right) m / z: 219\left(\mathrm{M}^{+}+\mathrm{NH}_{4}{ }^{+}\right)$.

\section{Isoxazole aldehyde 20}


Lithium aluminium hydride $(0.53 \mathrm{mmol})$ was added to a stirred solution of isoxazole 13 (entry-3, Table-1) $(0.43 \mathrm{mmol})$ in $5 \mathrm{~mL}$ of $\mathrm{THF}$ at $0{ }^{0} \mathrm{C}$. Reduction was completed within $15 \mathrm{~min}$. The mixture was hydrolyzed with a solution of potassium hydrogen sulfate $(0.74 \mathrm{mmol})$ in $3 \mathrm{~mL}$ of water. Then ether 10 $\mathrm{mL}$ was added, the aqueous phase was separated and extracted with ether. The organic phases were combined, washed with $3 \mathrm{~N} \mathrm{HCl}$, sat. $\mathrm{NaHCO}_{3}$, and sat. $\mathrm{NaCl}$ and dried with $\mathrm{Na}_{2} \mathrm{SO}_{4}$. Evaporation of the solvent gave crude aldehyde. ${ }^{1} \mathrm{H}$ NMR: $\left(300 \mathrm{MHz} \mathrm{CDCl}_{3}\right) \delta 10.10(1 \mathrm{H}, \mathrm{s}), 7.37-7.24(5 \mathrm{H}, \mathrm{m}), 6.32(1 \mathrm{H}$, s), $4.14(2 \mathrm{H}, \mathrm{s}) ;{ }^{13} \mathrm{C} \mathrm{NMR}:\left(75 \mathrm{MHz}, \mathrm{CDCl}_{3}\right) \delta 184.7,174.5,162.4,135.2,129.2,128.9,127.6,99.3$, 33.5. 

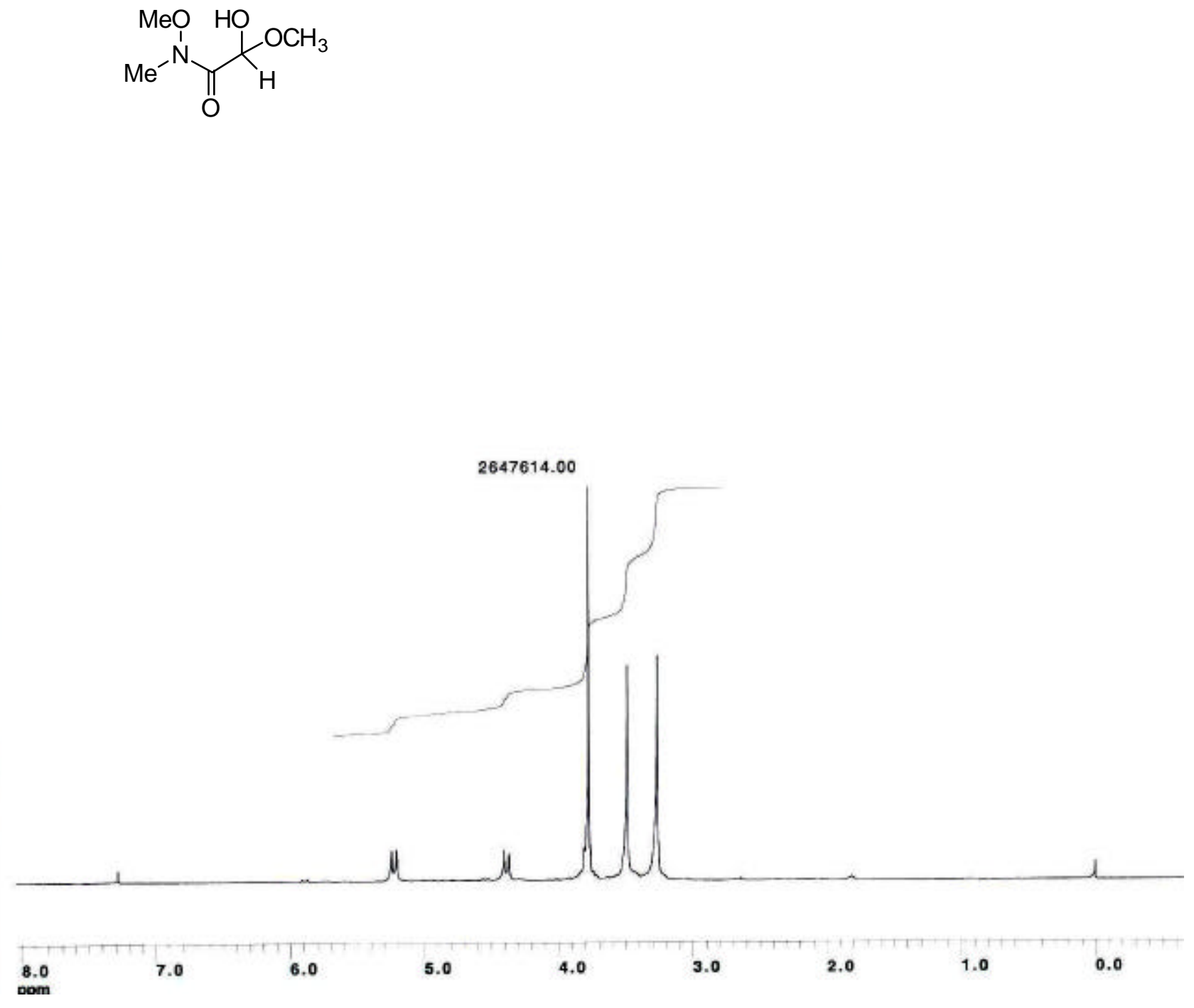

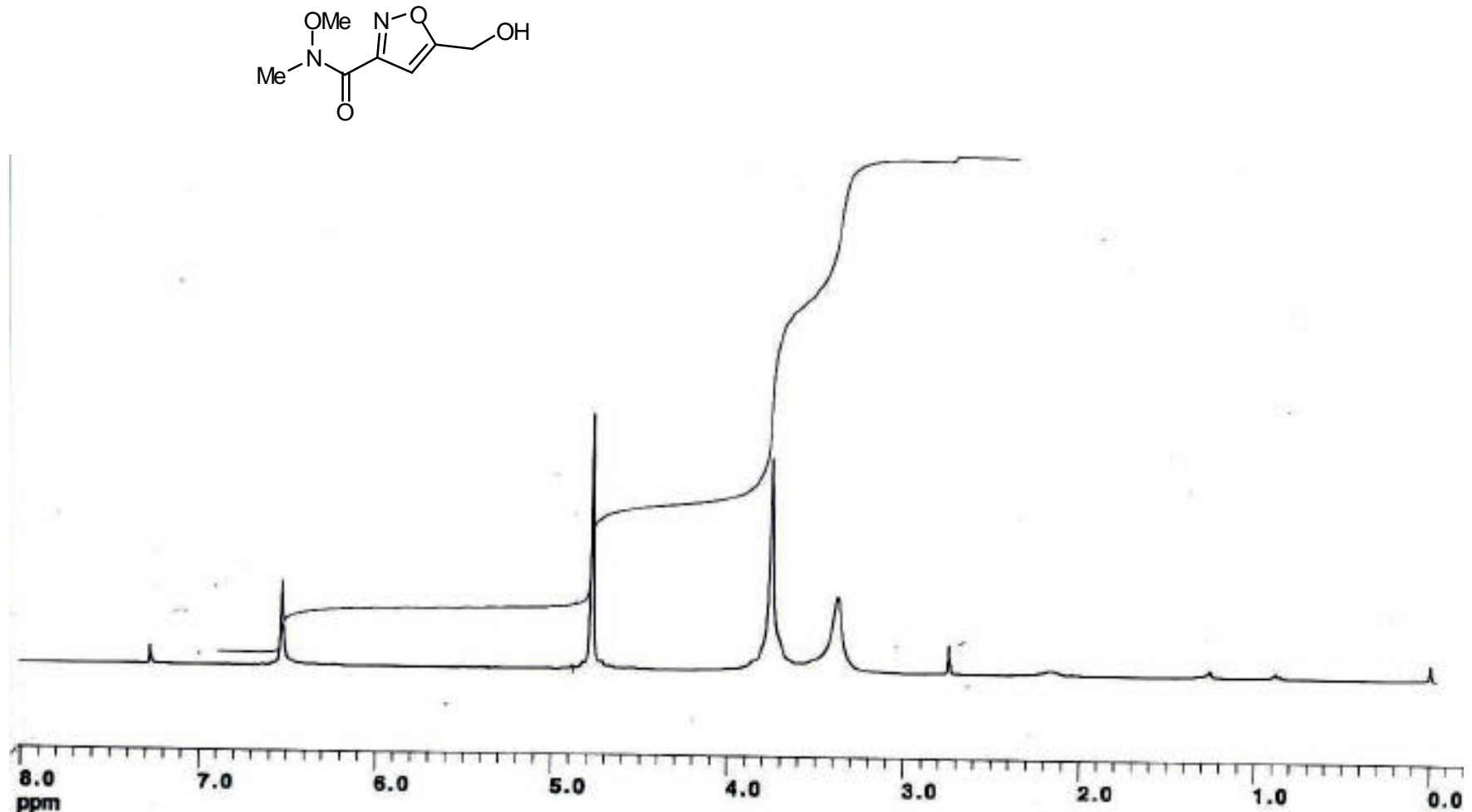
<smiles>CN(C)C(=O)c1cc(CO)on1</smiles>

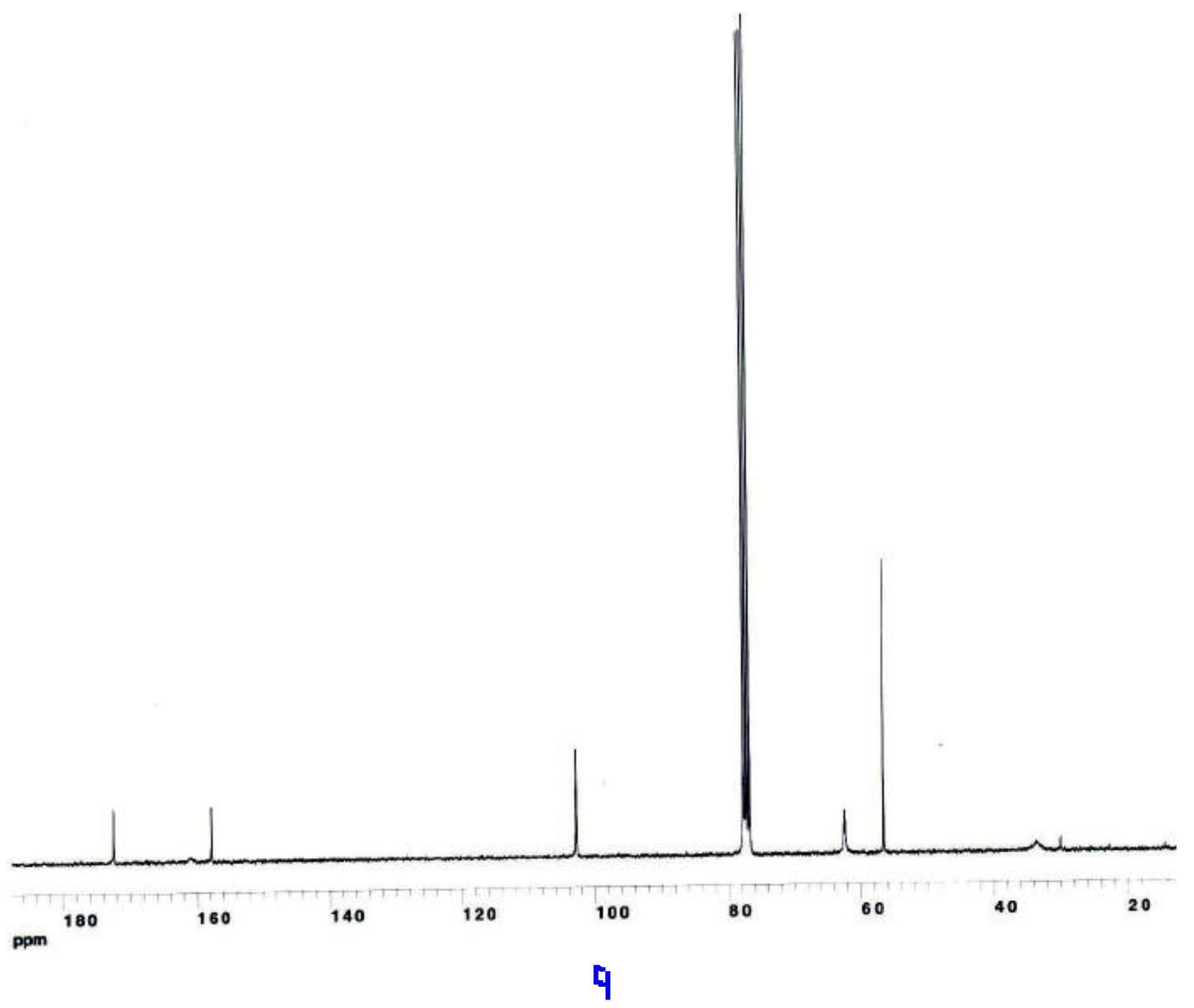


<smiles>CON(C)C(=O)c1cc(C(O)c2ccccc2)on1</smiles>

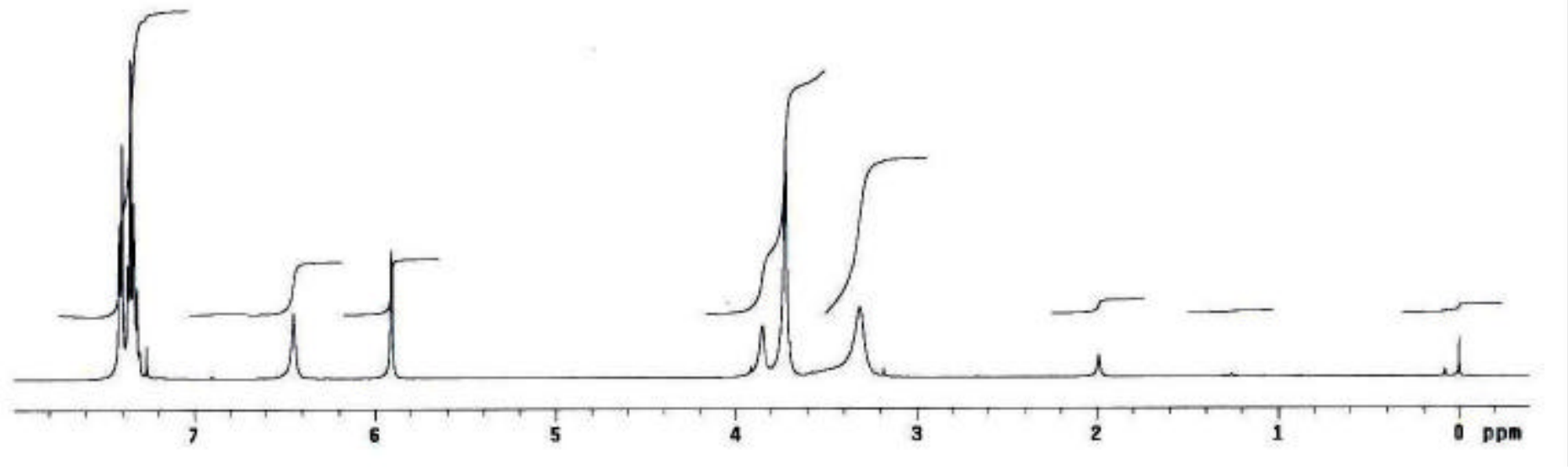


<smiles>CON(C)C(=O)c1cc(C(O)c2ccccc2)on1</smiles>

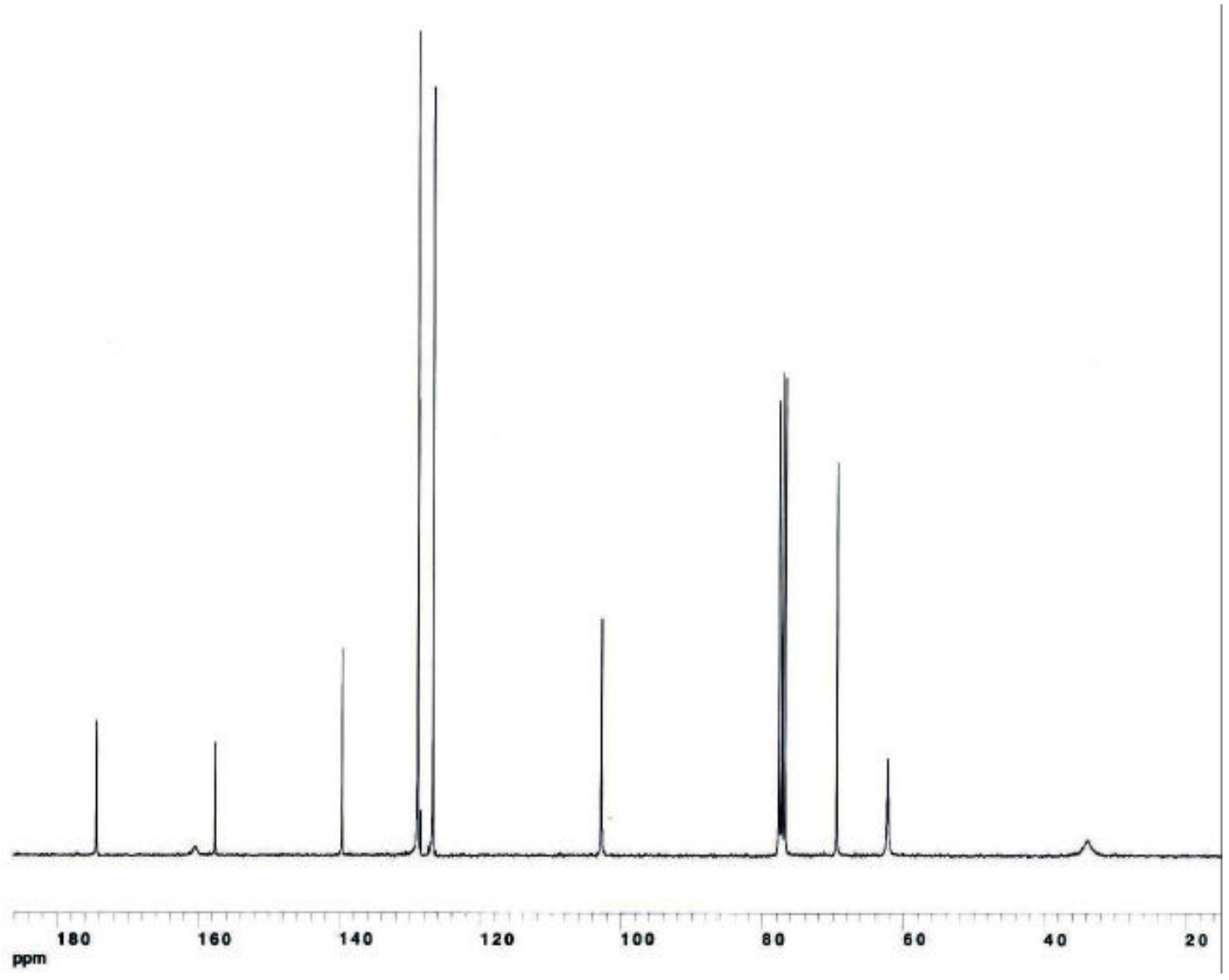




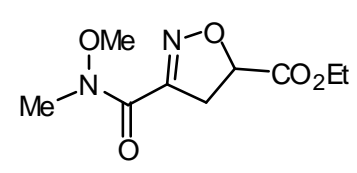

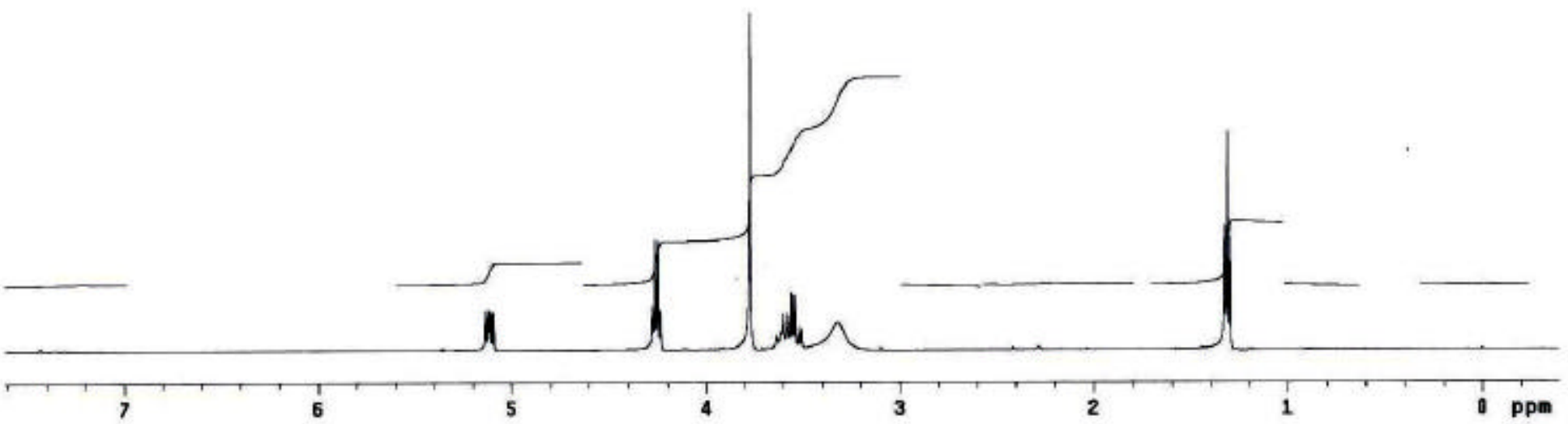


<smiles>CCOC1CC(C(=O)N(C)OC)=NO1</smiles>

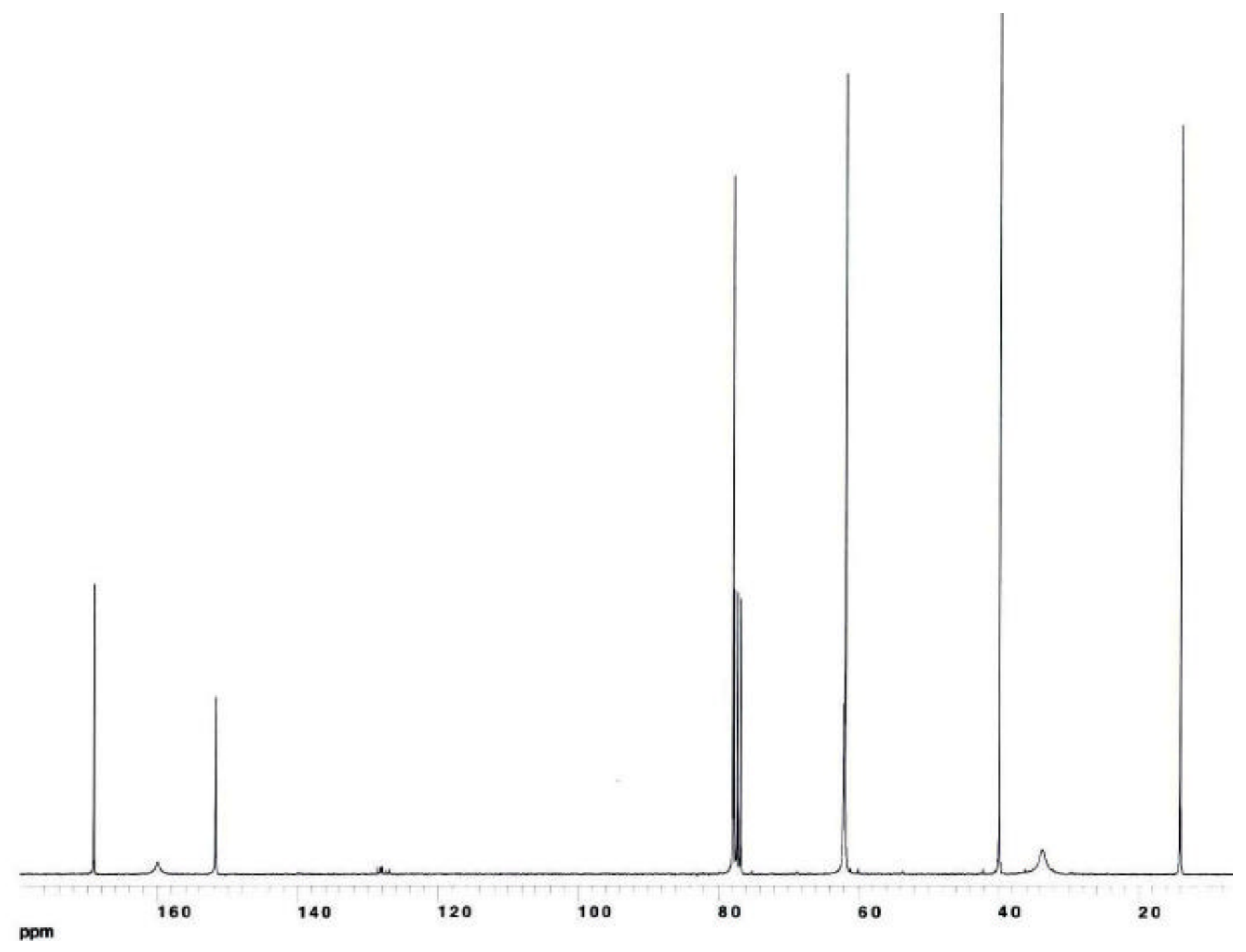



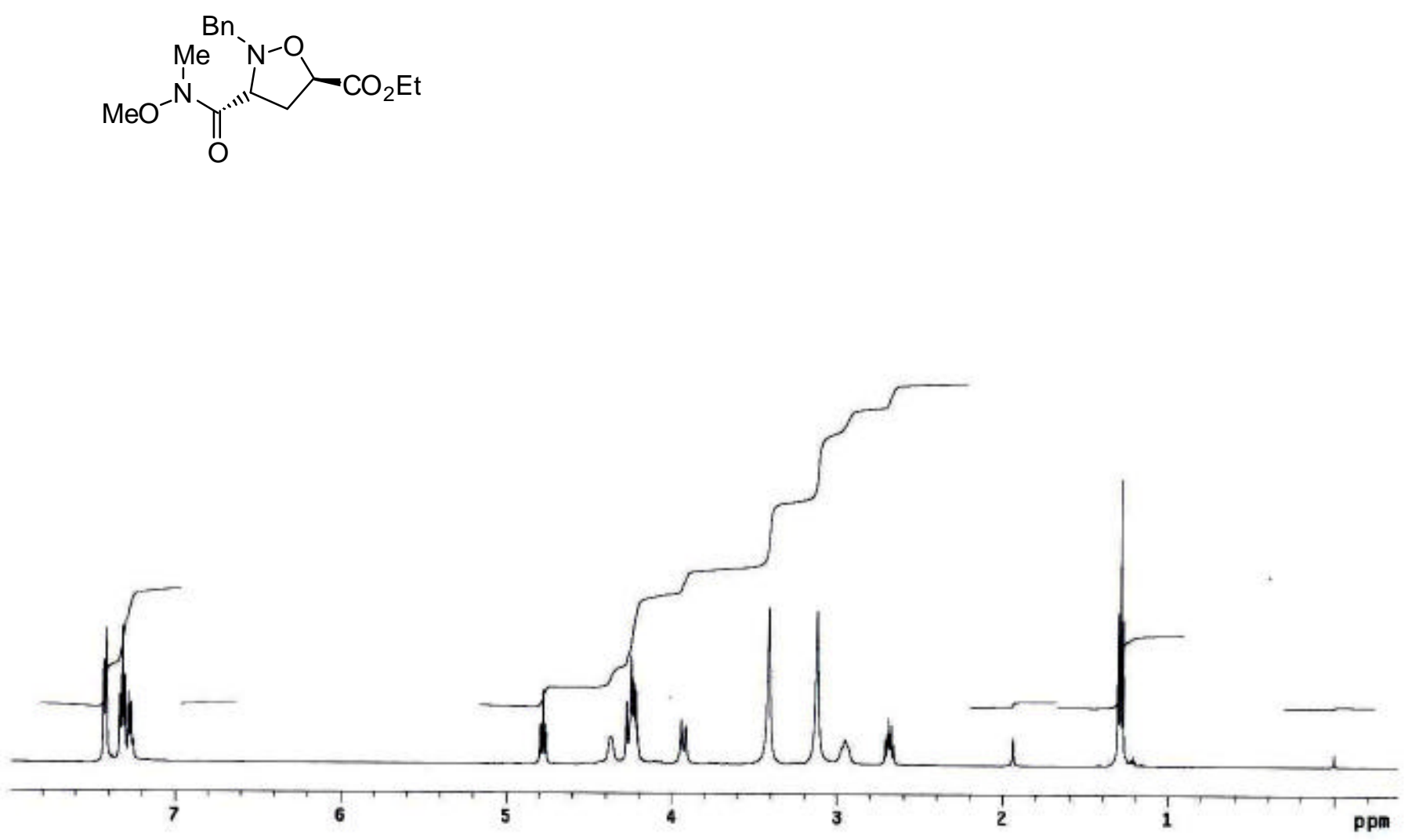

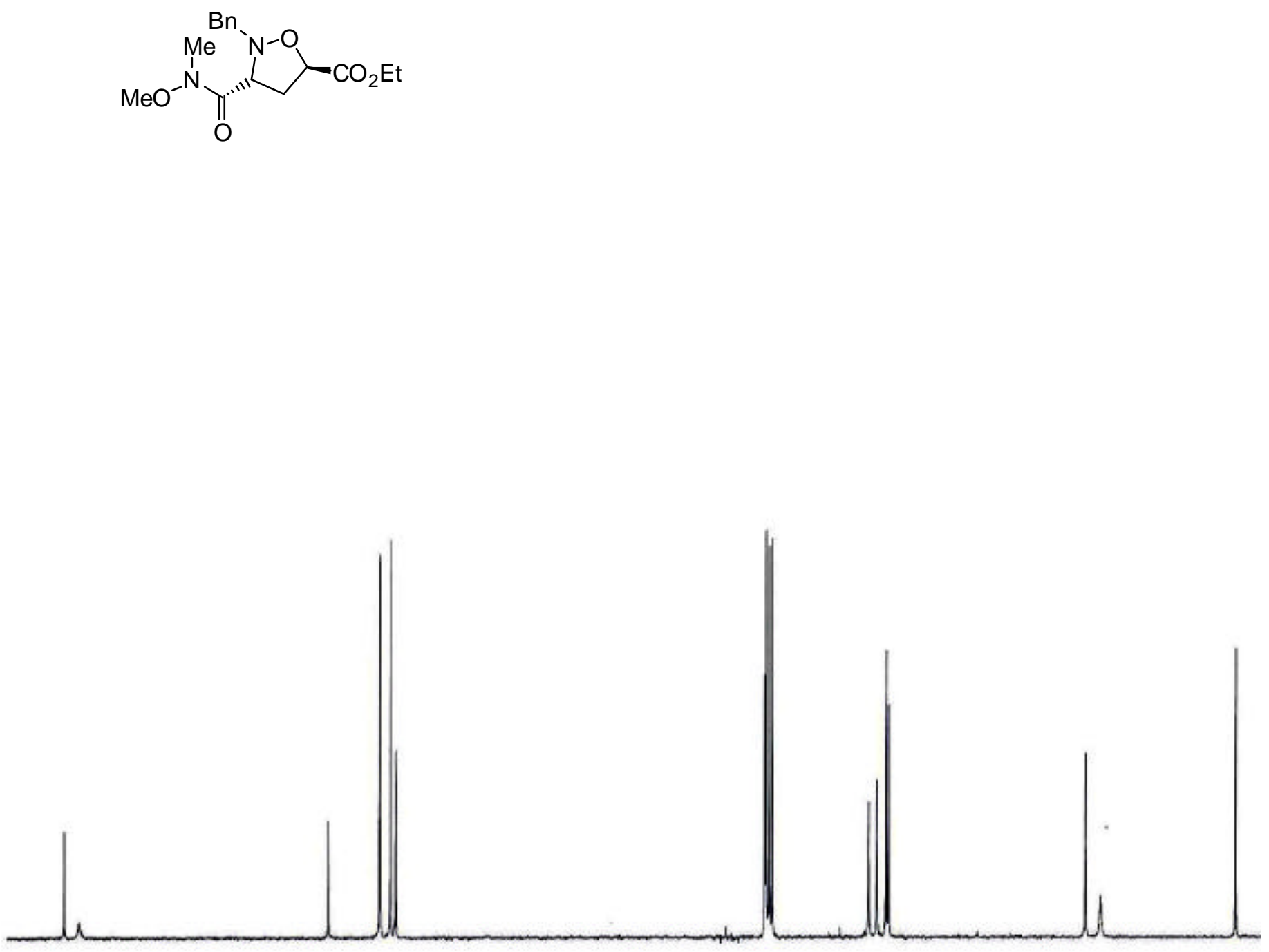

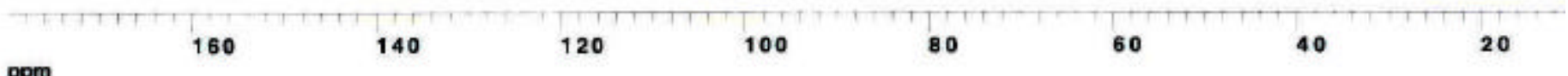


<smiles>CCOC(=O)C1C[C@@H](C(=O)N(C)OC)N(Cc2ccccc2)O1</smiles>

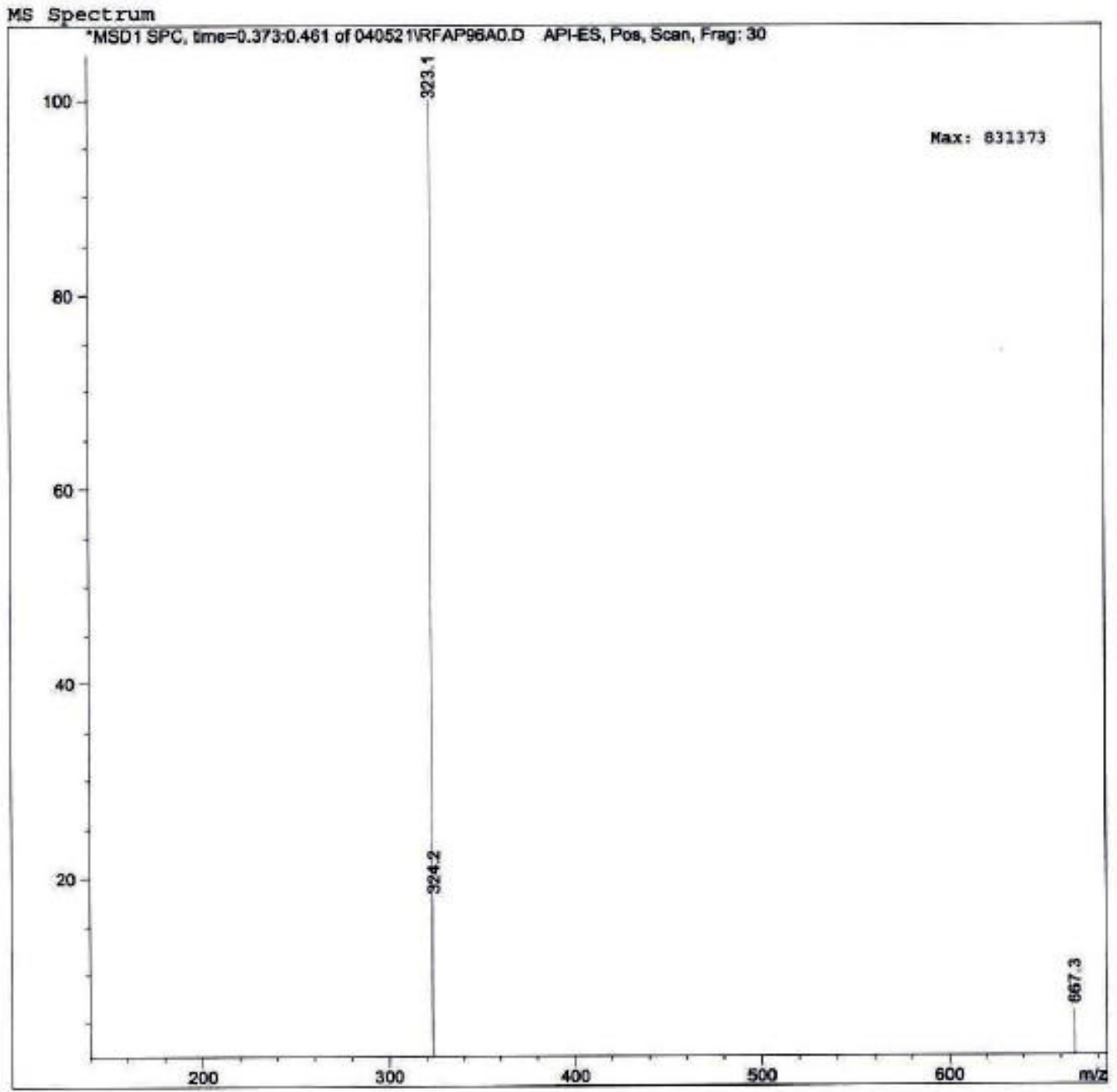


<smiles>CC(=O)c1cc(Cc2ccccc2)on1</smiles>

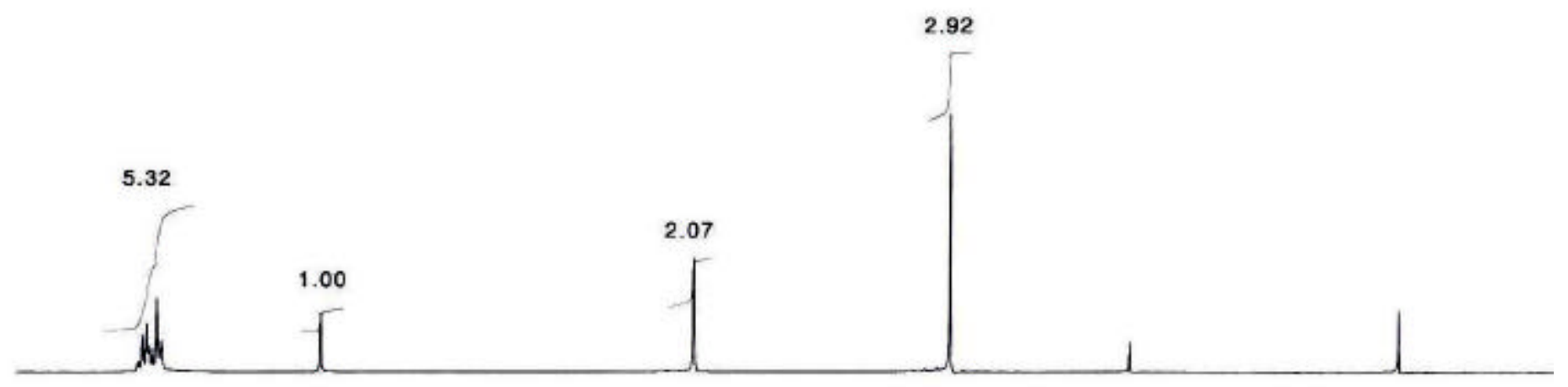

\begin{tabular}{|c|c|c|c|c|c|c|c|c|}
\hline $\begin{array}{r}8.0 \\
\mathrm{ppm}\end{array}$ & 7.0 & 6.0 & 5.0 & 4.0 & 3.0 & 2.0 & 1.0 & 0.0 \\
\hline
\end{tabular}


<smiles>CC(=O)c1cc(Cc2ccccc2)on1</smiles>

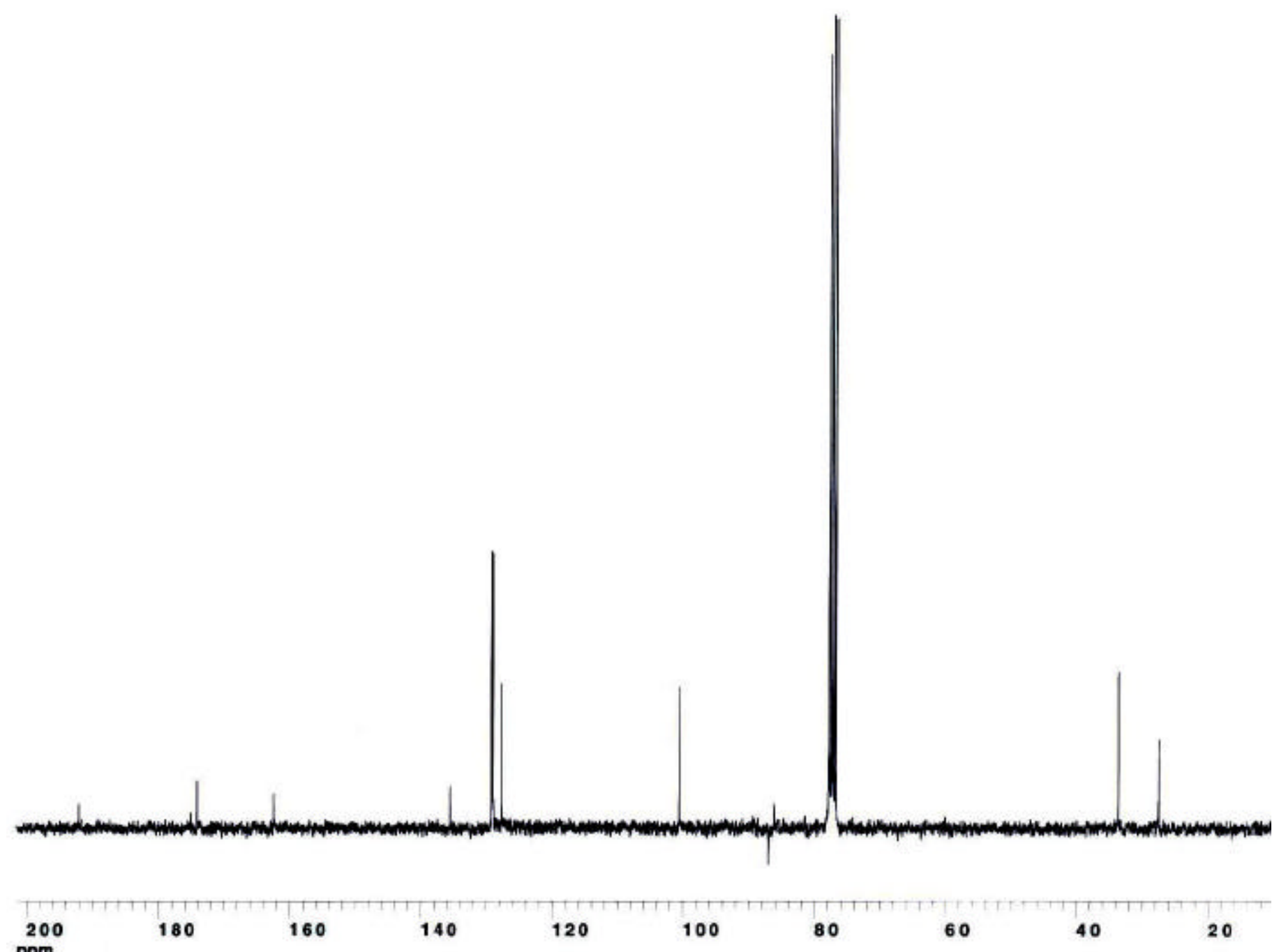


$\prod_{\mathrm{O}}$

MS Spectrum

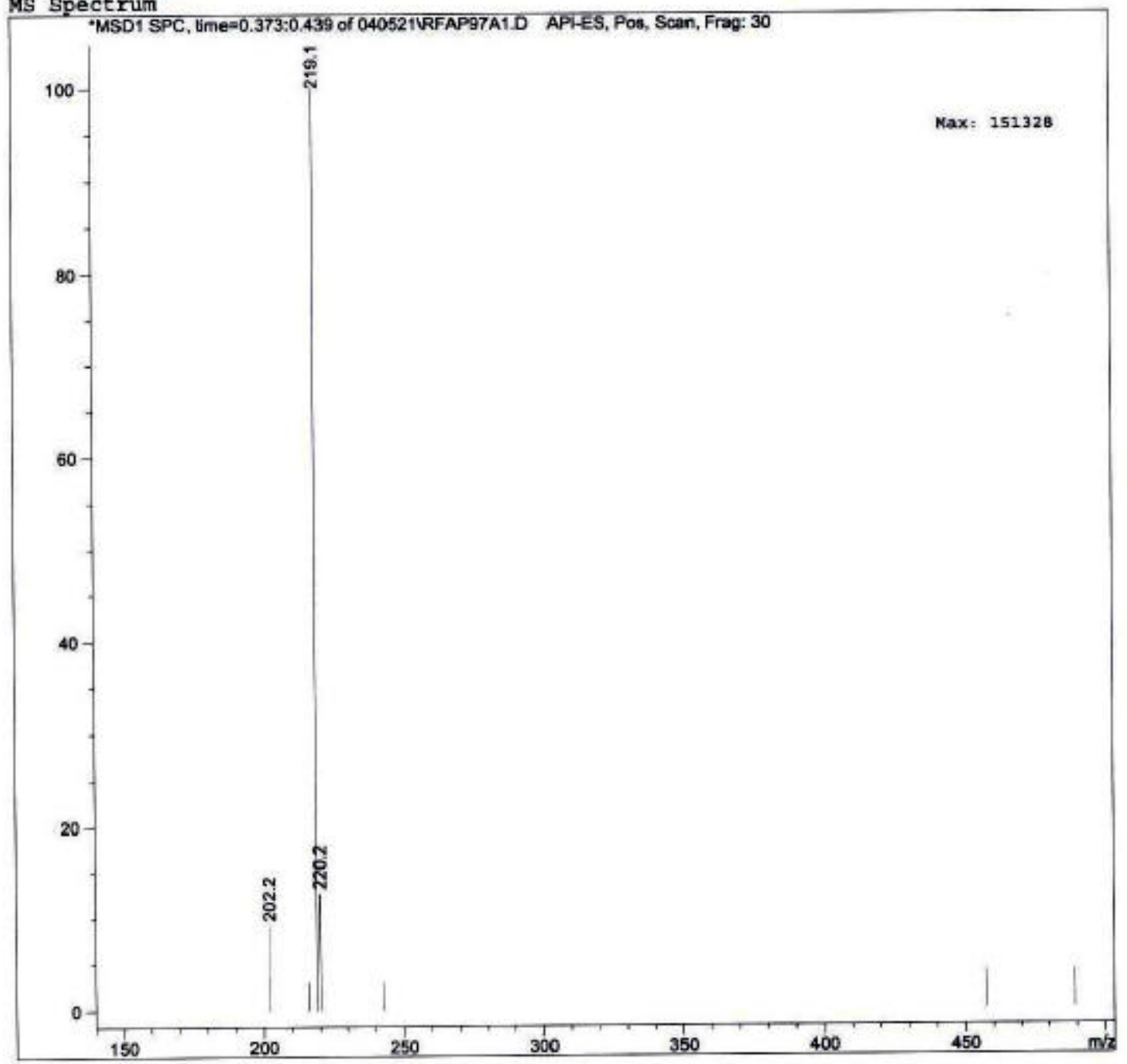


<smiles>O=Cc1cc(Cc2ccccc2)on1</smiles>

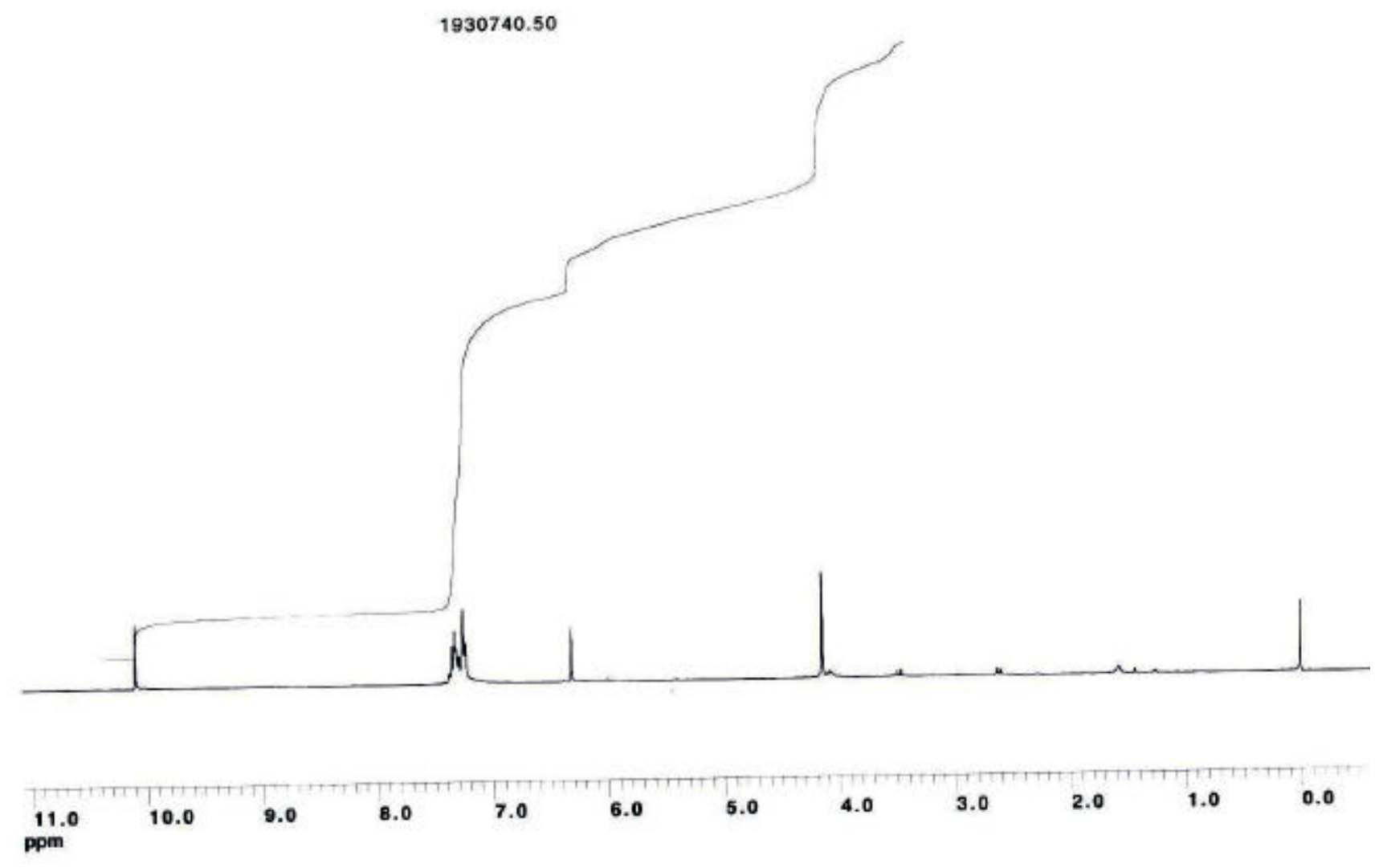

20 
<smiles>O=Cc1cc(Cc2ccccc2)on1</smiles>

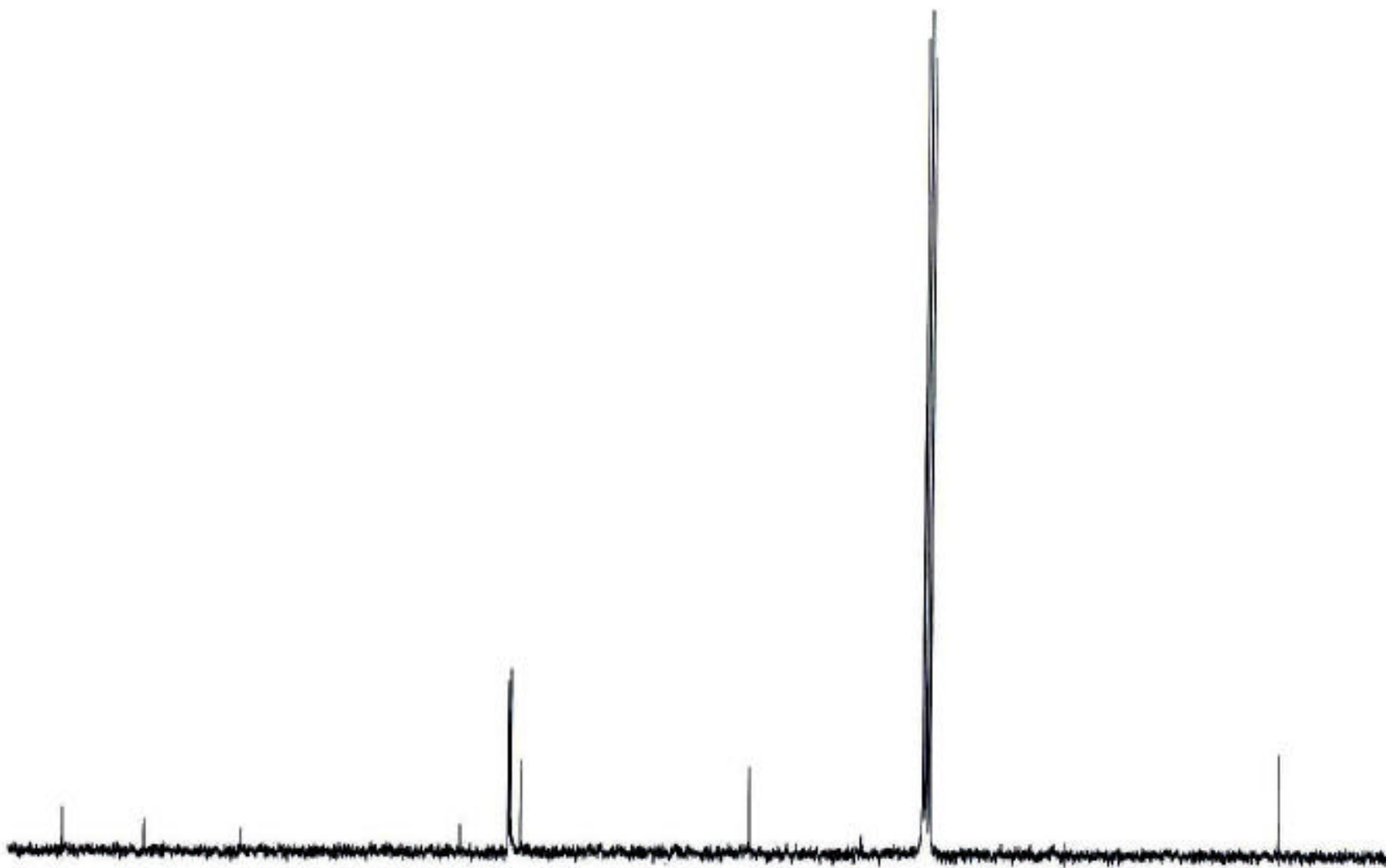

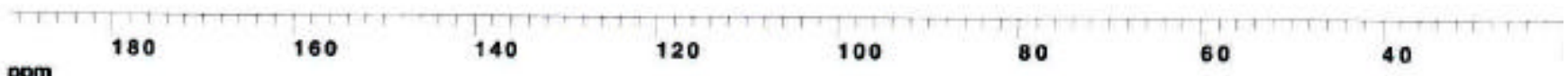

ppm 